\title{
Realistic constitutive relations for metal plasticity ${ }^{\text {is }}$
}

\author{
U.F. Kocks * \\ Center for Materials Science, Los Alamos National Laboratory, Los Alamos, NM 87545, USA
}

Received 3 May 2000

\begin{abstract}
The process of establishing quantitative constitutive relations is illustrated on the example of strain hardening: a phenomenological analysis in parallel with the derivation of physics-based combinations of variables; both followed by the use a sufficient set of experimental data to firm up unknown parameters or even unknown functions; and the eventual return to an assessment of the more detailed physical mechanisms that are suggested by the observed behavior. It is emphasized that a physics-based algebraic expansion around an accessible standard state is more reliable than a grand overall equation. (C) 2001 Elsevier Science B.V. All rights reserved.
\end{abstract}

Keywords: Modeling; Constitutive relations; Work hardening; Thermal activation; Dynamic recovery; Dislocation junctions; fcc; Polycrystals

\section{Introduction}

In the years leading up to 1975, Ali Argon, Mike Ashby and myself worked together on the 'Thermodynamics and Kinetics of Slip' [1], one of the most pleasant and most enlightening cooperative endeavors of my scientific career. This book dealt with the yield strength only or, in any case, with the behavior of materials at constant structure. In the same year, 1975, Ali Argon published a book he edited on 'Constitutive Equations in Plasticity' [2], to which the author also contributed [3], but in which many chapters dealt with changes in structure with straining. A central paper by Hart et al. [4] dealt with the problem of casting the effects of metallurgical structure in terms of the phenomenological concept of material state. Constitutive relations are meant to describe macroscopic material behavior - based, if possible, on the microscopic mechanisms underlying this behavior but, in any case, involving only macroscopically measurable parameters in the final equations and rules.

\footnotetext{
Dedicated to Ali Argon - to me a mentor, to materials science a pioneer.

* Tel.: + 1-505-6679323; fax: + 1-505-6652992.
}

The primary state parameter introduced in [1] is the mechanical threshold $\hat{\tau}$, the extrapolated flow stress at absolute zero temperature, i.e. in the absence of thermal activation. It marks the limit above which dislocations cannot find (in the average) any position of mechanical equilibrium in any slip plane and above which, therefore, dynamic effects govern the behavior. Over most of the regime of practical interest, on the other hand, thermal activation of dislocations over obstacles governs the kinetics of plasticity and the flow stress is below the mechanical threshold. When there is more than one type of obstacle or mechanism, $\hat{\tau}$ may be composed of multiple terms, each of them a state parameter, but the total $\hat{\tau}$ remains pre-eminent.

When the state changes, for example by work hardening during a strain increment $\mathrm{d} \varepsilon$, this is reflected in a change $\mathrm{d} \hat{\tau} / \mathrm{d} \varepsilon$. It turns out that this strain-hardening rate is itself strongly dependent on the temperature and strain rate at which the straining was performed, owing to the phenomenon of dynamic recovery. We will use this thermally activated hardening mechanism as a prime example of constitutive-relations development in the present paper. The materials to which it will be applied are fcc polycrystals, for which we have the best data; but the principles hold for many other cases. 


\section{The phenomenology of strain hardening}

Fig. 1 shows a subset of stress-strain curves for copper polycrystals over a wide range of temperatures and strain rates [5]. A number of properties are immediately evident in a qualitative way, the variation in the yield stress with temperature and strain rate is much less than that of the curves as a whole; the initial hardening rate is also similar in all curves; and finally, all curves seem to consist of a single-parameter set. We shall show that indeed a single stress-scaling parameter can be found to unify the description adequately, and that this parameter alone governs the temperature and rate dependence of strain hardening.

A proper description of hardening [6] is a plot of the hardening rate, $\Theta \equiv \mathrm{d} \sigma / \mathrm{d} \varepsilon$, versus the stress, $\sigma$ (here the flow stress in a compression test); this is shown in Fig. 2 for the same set of curves as Fig. 1. Fig. 3 replots the same data in a scaled form, $\Theta$ is divided by the shear modulus (at the respective temperatures); $\sigma$ is scaled by a parameter $\sigma_{\mathrm{v}}$, picked for best fit for each curve. This parameter could have been chosen in many ways; for example, the stress value in the mid-range [7], or (as in Fig. 3) the extrapolation to $\Theta=0$ of the more-or-less linearly decaying portion. The latter is called $\sigma_{\mathrm{v}}$ because, if the curves actually decayed as $\left(1-\sigma / \sigma_{\mathrm{v}}\right)$, the Voce law would hold [8]. In Section 4 below, we will describe the dependence of $\sigma_{\mathrm{v}}$ on temperature and strain rate. We will discuss further down what physical meaning it may have.

Parenthetically we note that strain hardening in polycrystals is also influenced by texture changes during deformation. In the original paper [5], we simulated these by LApp (the Los Almos polycrystal plasticity code, see [9]), after postulating a unique grain-level hardening law $\theta(\tau)$ - which was altered until a sufficient subset of the polycrystal data were matched. It is the Voce stress $\tau_{\mathrm{v}}$ of this local law that was actually used to derive the thermal activation behavior. In the following, we will continue to discuss $\sigma_{\mathrm{v}}$ (the macroscopic value for the compression test) rather than $\tau_{\mathrm{v}}$, for convenience. In addition, we are using the value of the Voce stress at the test temperature, rather than that determined by subsequent probes to obtain $\hat{\sigma}_{\mathrm{v}}$, the latter would be more proper since it is the change in state that strain hardening is supposed to characterize [10]; but the former, much simpler procedure, is allowable because the rate and temperature dependence at constant state is so slight.

\section{Thermal activation in plasticity}

The strain rate connected with the thermally activated overcoming of obstacles can be written as

$$
\dot{\varepsilon}=\dot{\varepsilon}_{0} \exp -\frac{\Delta G(\tau / \hat{\tau})}{k T}
$$

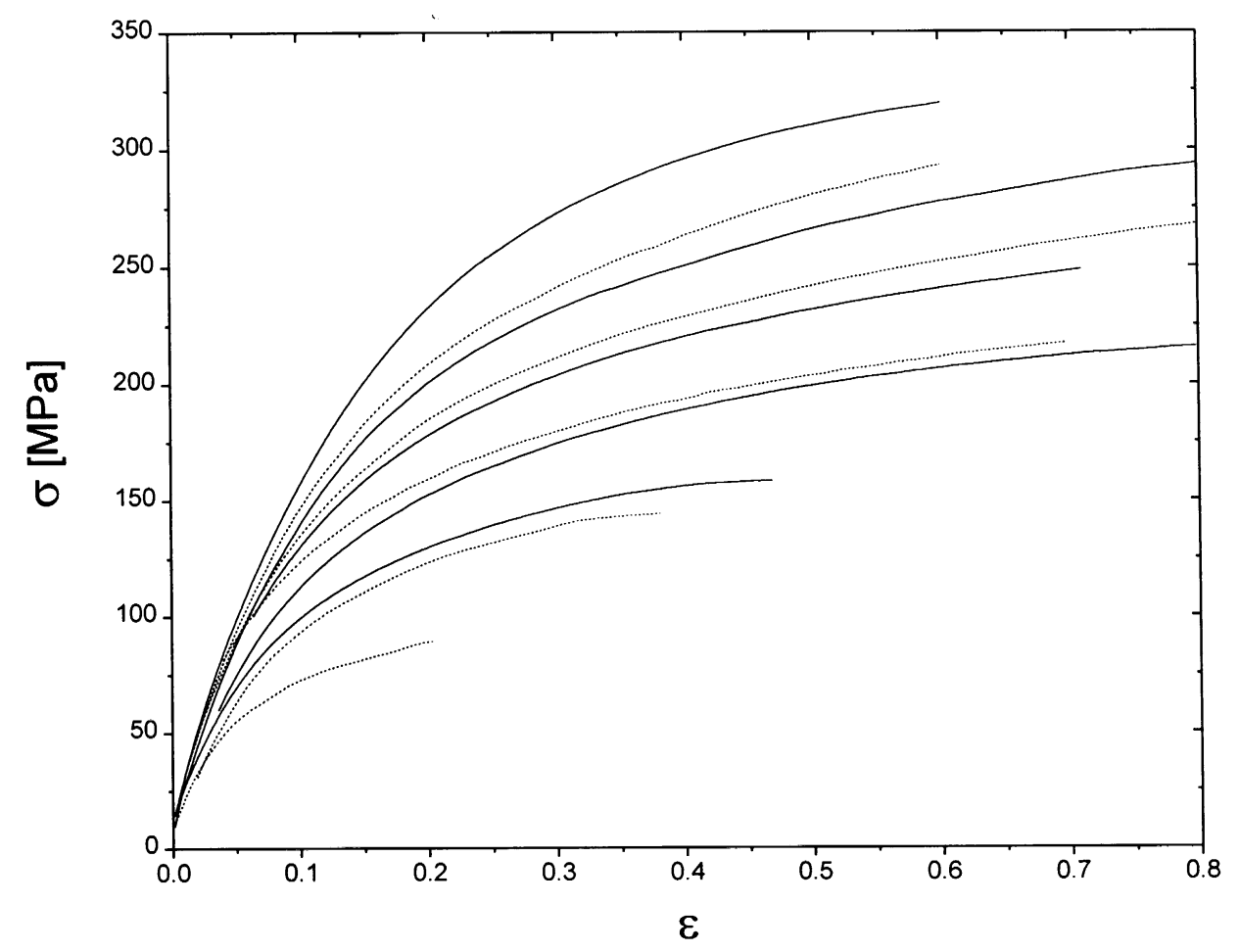

Fig. 1. Stress-strain curves of compressed OFE copper polycrystals (grain size $\approx 25 \mu \mathrm{m}$ ). Strain rates $10^{-1} \mathrm{~s}^{-1}$ (solid lines) and $10^{-4} \mathrm{~s}^{-1}$ (dashed). Room temperature and 100, 200, 300, $400 \mathrm{C}$ (in sequence) [5]. 


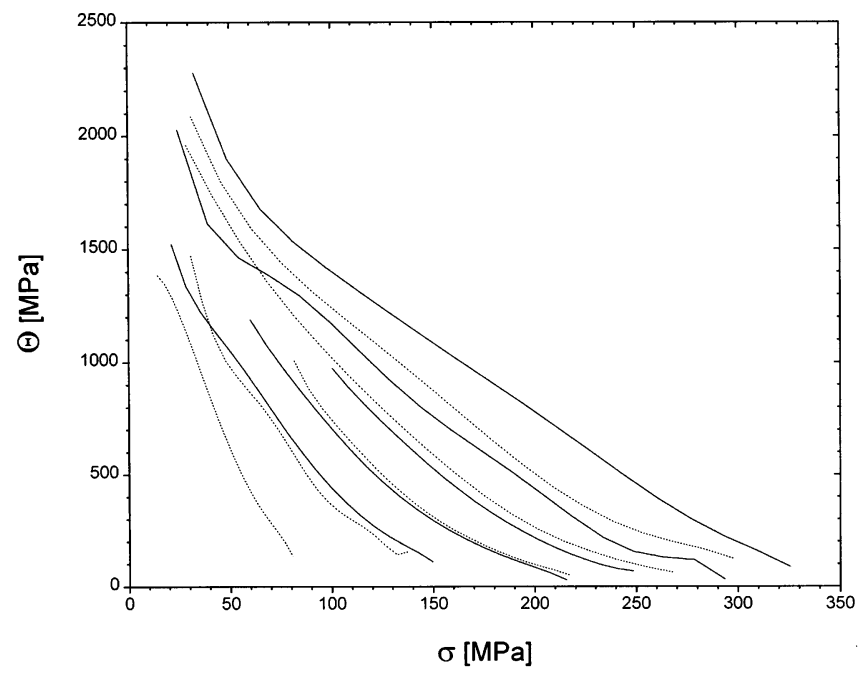

Fig. 2. Same data as Fig. 1, plotted as strain-hardening rate $\Theta$ versus stress $\sigma$.
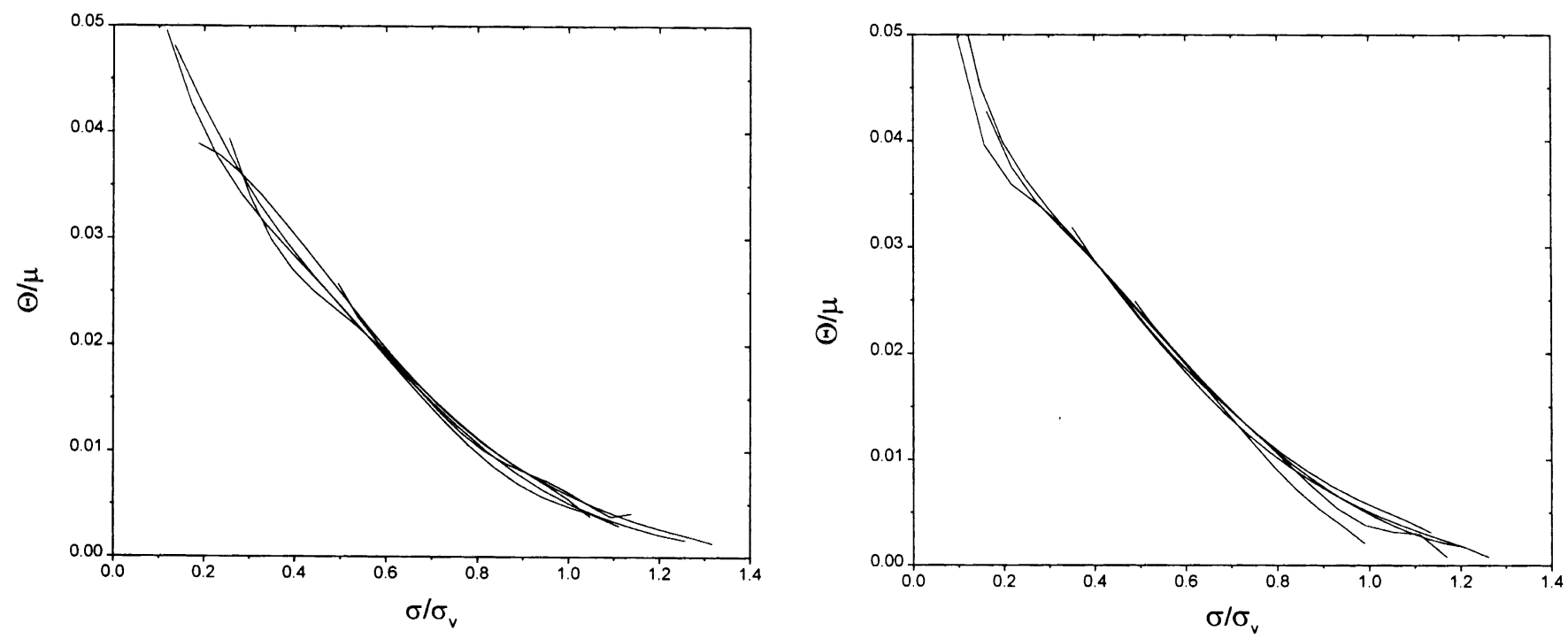

Fig. 3. Same data as Fig. 2, scaled by the temperature-dependent shear modulus $\mu$ on the ordinate, and by a heuristic value $\sigma_{\mathrm{v}}$ for each curve on the abscissa. (a) $10^{-4}$, (b) $10^{-1} \mathrm{~s}^{-1}$.

where the 'activation energy' (activation free enthalpy) $\Delta G$ is a function of the (locally) applied stress $\tau$ and the 'height' of the obstacle $\hat{\tau}$ (the stress where $\Delta G=0$ ). The preexponential term is determined by the attempt frequency, the number of places per unit volume at which an attempt is made, and the strain achieved with each successful thermal activation. A dependence on stress and temperature of $\dot{\varepsilon}_{0}$ is almost always negligible with respect to the explicit dependencies in the exponent [1]. A sensible order of magnitude for $\dot{\varepsilon}_{0}$ can be anywhere between $10^{5}$ and $10^{10} \mathrm{~s}^{-1}$.
A slight but important complication is that the flow stress at a given temperature, $T$, is typically proportional to an appropriate shear modulus, $\mu$, at this temperature. In order for the activation work done by the applied forces during the activation event to be independent of material properties, it is necessary to have $\Delta G$ be proportional to $\mu(T)$ also (which is a special but reasonable way to incorporate entropy effects into the free energy of activation [1]). For dimensional reasons, one sets $\Delta G \propto \mu b^{3}$. One can then define a normalized activation energy gas 
$g \equiv \frac{\Delta G}{\mu b^{3}}=g\left(\frac{\tau}{\mu} \frac{\mu_{0}}{\hat{\tau}}\right)$

where $\mu_{0}$ is the shear modulus at $0 \mathrm{~K}$. When the function is inverted, one obtains

$\frac{\tau}{\mu}=\frac{\hat{\tau}}{\mu_{0}} s\left(\frac{g}{g_{0}}\right)$

where a scaling factor $g_{0}$ has been introduced which characterizes the activation energy in the extrapolation to $\tau=0$.

As it enters into Eq. (3), the normalized activation energy should be expressed in terms of temperature and strain rate, by inversion of Eq. (1):

$g=\frac{k T}{\mu b^{3}} \ln \left(\frac{\dot{\varepsilon}_{0}}{\dot{\varepsilon}}\right)$

Eqs. (3) and (4) couple the $T$ - and $\dot{\varepsilon}$-dependencies of the flow stress - if the parameter $\dot{\varepsilon}_{0}$ is known. A useful procedure is to attempt to find an $\dot{\varepsilon}_{0}$-value empirically, such that it does unify the $T$ - and $\dot{\varepsilon}$-dependencies and hope that it comes out in a physically reasonable order of magnitude. This procedure was used in Fig. 4 for the scaling-stress $\sigma_{\mathrm{v}}$ from all the data in [5], of which a subset was used in Figs. 1-3. It is seen that the very satisfactory value of $\dot{\varepsilon}_{0}=10^{7} \mathrm{~s}^{-1}$ did indeed achieve such a unification, with considerable regions of overlap. A value $1 / 3$ or 3 times this would not have worked; and neglecting the $T$-dependence of $\mu$ would have given quite a different value.

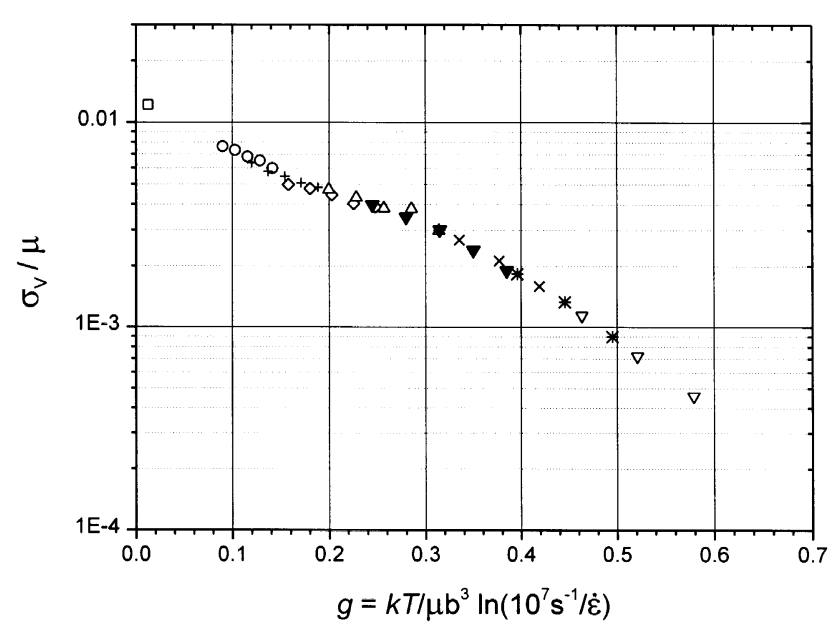

Fig. 4. The stress scaling factor $\sigma_{\mathrm{v}}$ as determined from plots of the kind of Fig. 3 for all the data in [5], at 5 strain rates and 5 temperatures, plotted logarithmically versus the normalized activation energy, using $\dot{\varepsilon}_{0}=10^{7} \mathrm{~s}^{-1}$ in order to make all points fall on a single curve.

\section{Constitutive descriptions for dynamic recovery}

We have seen that dynamic recovery, the $T$ - and $\dot{\varepsilon}$-dependent decay of the strain hardening rate, can be characterized by the Voce stress $\sigma_{\mathrm{v}}$, as it was plotted in Fig. 4 (divided by $\mu$ ) against the normalized activation energy. Let us now discuss how a constitutive relation for this $T$ - and $\dot{\varepsilon}$-dependence can be obtained. We will continue to write the fundamental expressions in terms of the glide resistance, $\tau$, and use $\sigma_{\mathrm{v}}$ only for the scaling parameter in the hardening experiments.

The plot in Fig. 4 was chosen such that it would have given a straight line if the activation energy $\Delta G$ were proportional to the logarithm of $\sigma_{\mathrm{vo}} / \sigma$ (where $\sigma_{\mathrm{vo}}$ is the stress value at $\Delta G=0$ ). This 'law' has been widely used for dynamic recovery (albeit as characterized by a starting stress $\tau_{\text {III }}[11,12]$, and it was also suggested in [1] as an approximation that might hold over a relatively large range. It leads directly to a power law between stress and strain rate. It is obviously not obeyed over the entire range of experiments shown in Fig. 4, since the line is not straight. We have argued [13] that there is no reason to expect that any law for such a complex process will ever be derived from first principles; perhaps a nomographic form such as in the empirical diagram Fig. 4 is the best venue for an accurate description.

We would like to suggest here that an expansion around the behavior under a chosen standard set of conditions may serve well. This has the additional advantage that the 'standard' flow stress can now serve as a state parameter, rather than that extrapolated to zero temperature, which is awkward to measure. If we mark the standard conditions with the subscript 1 , we have, instead of Eq. (3),

$\frac{\tau}{\mu}=\frac{\tau_{1}}{\mu_{1}} \frac{s\left(g / g_{0}\right)}{s_{1}\left(g_{1} / g_{0}\right)}$

and an expansion according to a logarithmic law would give the local power law

$\frac{\dot{\varepsilon}}{\dot{\varepsilon}_{1}}=\left(\frac{\tau}{\mu} \frac{\mu_{1}}{\tau_{1}}\right)^{n_{1}}$

or the derivative form

$\left.\frac{\partial \ln \tau}{\partial \ln \dot{\varepsilon}}\right|_{T_{1}} \equiv \frac{1}{n_{1}}=\frac{k T_{1}}{A_{1} \mu_{1} b^{3}}$

where $A$ is the slope indicated in Fig. 4, and the stress exponent $n$ is proportional to $1 / T$ - a direct consequence of any stress-dependent activation energy. In a similar way, neighboring temperatures can be covered by the derivative

$-\left.\frac{\partial \ln (\tau / \mu)}{\partial \ln (T / \mu)}\right|_{\dot{\varepsilon}_{1}}=\frac{g_{1}}{A_{1}}=\frac{\ln \left(\dot{\varepsilon}_{0} / \dot{\varepsilon}_{1}\right)}{n_{1}}$ 


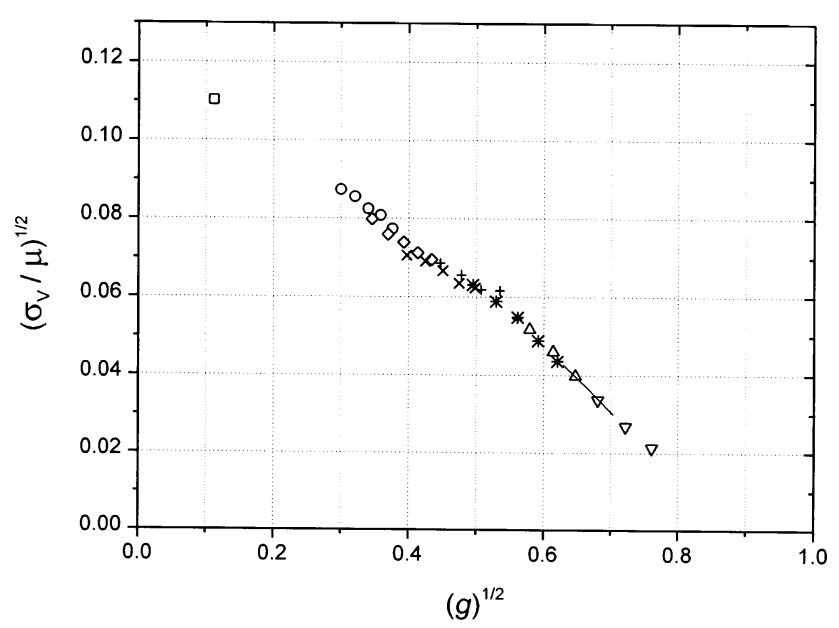

Fig. 5. Same data as Fig. 4, plotted according to Eq. (9).
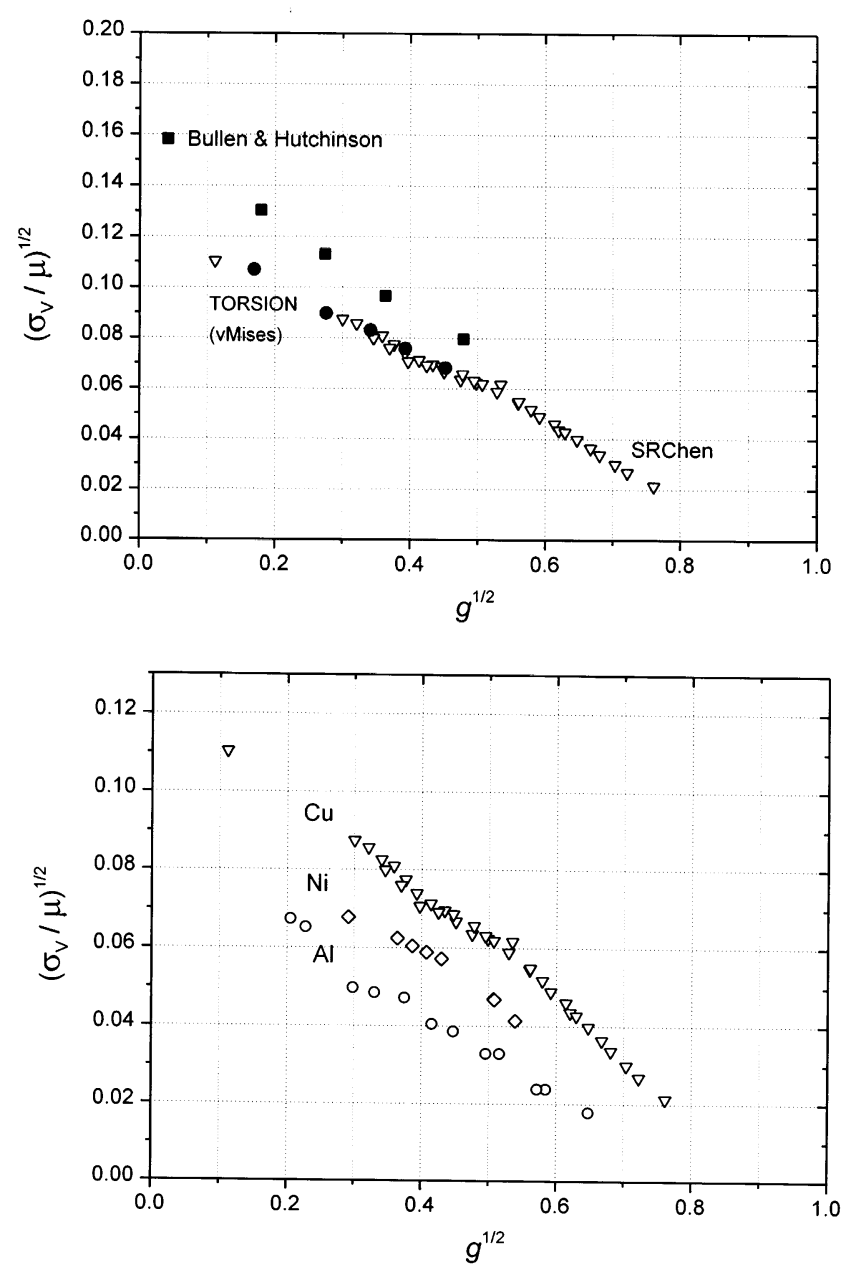

Fig. 6. Similar plot as Fig. 5, (a) for different tests on copper; (b) for three fcc polycrystalline materials.

Eqs. (7) and (8) may well suffice to describe the range of strain rates and temperatures encountered in an engineering body (which may be subject to adiabatic heating). They must be complemented by a measure- ment of the state parameter $\tau_{1}$, for which the standard conditions are best chosen as a relatively low temperature in the regime of interest (so there is least chance for recovery) and a strain rate of about $10^{-3} \mathrm{~s}^{-1}$ (because it is slow enough to avoid adiabatic heating - and to allow real-time observation - and fast enough to expect little fluctuation in temperature).

We wish to point out one further advantage of this representation. The coefficient $A$ is the normalized activation work which is, like the activation energy, a local property of the obstacle, not dependent on the obstacle density (and distribution) as the flow stress and the 'activation volume' are (in opposite ways) [1].

The quest for a closed-form description has nevertheless continued [14], and we can report here, for the first time, that the relation

$g=g_{0}\left[1-\left(\frac{\sigma_{\mathrm{v}} / \mu}{\sigma_{\mathrm{vo}} / \mu_{0}}\right)^{1 / 2}\right]^{2}$

does in fact fit the data in Fig. 4 quite well; this is shown in Fig. 5. The small 'hump' in the mid-range could perhaps be due to a small amount of dynamic strain aging, owing to even a very low concentration of solutes. Fig. 6a and b show it applied to other tests in $\mathrm{Cu}$ and to some other materials, respectively. It appears (quite sensibly) that the 'mechanical' Voce stress, $\sigma_{\mathrm{vo}}$, may depend on the specimen or test series, but the normalized activation energy, $g_{0}$, is a material property - which, in Fig. 6b, is seen to be in the correct sequence for the normalized stacking-fault energy of these materials.

If Eq. (9) holds, at least locally, then the expansion coefficients in Eqs. (7) and (8) become

$-\left.\frac{\partial \ln (\tau / \mu)}{\partial \ln (T / \mu)}\right|_{\dot{\varepsilon}_{1}}=\frac{\ln \left(\dot{\varepsilon}_{0} / \dot{\varepsilon}_{1}\right)}{n_{1}}=\frac{1}{\sqrt{g_{1} / g_{0}}-1}$

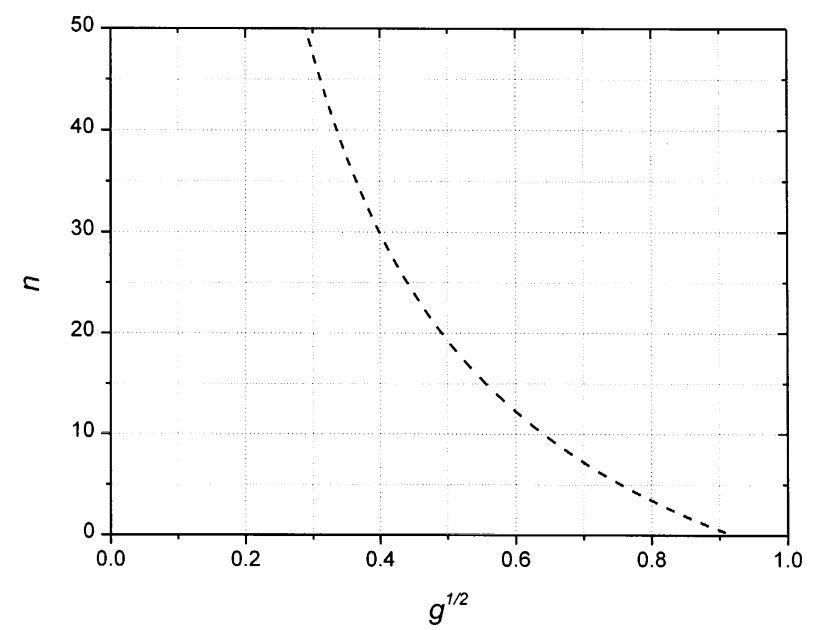

Fig. 7. The stress exponent $n$ to be used in an expansion around a local condition of temperature and strain-rate specified by the combination in $g$, as derived from Eq. (9). 


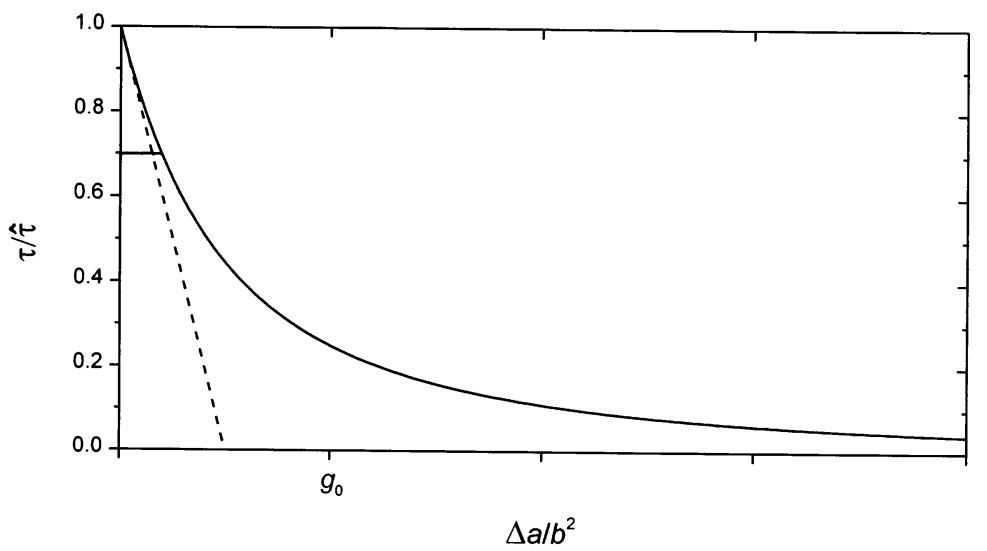

Fig. 8. The obstacle profile for the flow stress $\tau$ as a function of the activation area $\Delta a$, as derived from Eq. (9). In dynamic recovery processes, the obstacles inside the cell walls are pre-loaded by the local forward internal stress $0.7 \hat{\tau}$ and the applied stress is added to this.

The resulting function $n(\mathrm{~g})$ is plotted in Fig. 7 for $\dot{\varepsilon}_{1}=10^{-3}$ and $\dot{\varepsilon}_{0}=10^{7} \mathrm{~s}^{-1}$.

Eq. (9) is a special form of the phenomenological equation proposed in [1] and used in the MTS model [10] (for all processes other than dynamic recovery):

$g=g_{0}\left[1-\left(\frac{\tau / \mu}{\hat{\tau} / \mu_{0}}\right)^{p}\right]^{q}$

The exponent $q$ has been fixed to $q=2$ for dynamic recovery processes, by the experiments leading to Eq. (9), and should be general for all dislocation-forest interactions; it corresponds to a peaked obstacle (see below). The exponent $p=1 / 2$ characterizes the tail of the obstacle and is not as certain; unfortunately, the value of $g_{0}$ is sensitive to the choice of $p$. Actually, $g_{0}$ does not have much physical meaning, since it refers to a state that is never reached $(\tau=0)$ in longer-range obstacles. The values of $g_{0}$ derived here are, correspondingly, quite high. Of course, it is still true that this parameter, together with $\hat{\tau}$ (or $\sigma_{\mathrm{vo}}$ ), determines the slope in Figs. 5 and 6 .

\section{Dislocation forest interaction}

It is well established (see, for example, Kocks [15]) that the flow stress contribution due to dislocation interactions is controlled by forest intersections and that, among these, the breaking of attractive junctions is likely to be predominant [16]. There are many of these, characterized by various intersection angles, etc., so that an average interaction profile of some generality is not likely to ever be derived on the basis of first principles. If we, then, treat these obstacles as having a single phenomenological profile such as that described by Eq. (11), we would want to use experiments to determine the four parameters $p$, $q, \hat{\tau}$, and $g_{0}$. This requires flow stress measurements over a wide range of temperatures and strain rates (which then fix, to sufficient accuracy, the parameter $\dot{\varepsilon}_{0}$ in Eq. (4)).
The trouble with this procedure is that the range of stresses covered would still be only about the top $20 \%$ of the profile-because the obstacles are relatively 'athermal'. Note that this does not justify the addition of an athermal plateau stress [16], because the Cottrell-Stokes law is obeyed for this case [17]. The profile is expected to have a long tail, meaning a low value of $p$ and a very large value of $g_{0}$.

There is no mechanism of dynamic recovery that is generally accepted (so far). We believe [14] that it, too, is controlled by the breaking of attractive junctions namely those inside tangles and (diffuse) cell walls. They were not overcome during the slip process (in part because they were topologically isolated [15]) but, on the other hand, they are under a strong forward internal stress (because the tangles and cell walls are polarized). This internal stress, together with the applied stress, can lead to overcoming of these obstacles even at absolute zero temperature (where dynamic recovery is observed); a combination with thermal activation leads to the strong temperature and strain-rate dependence of the (extrapolated) saturation stress, at which dynamic recovery and athermal storage would balance.

The foregoing analysis has led to an experimental determination of the effective obstacle profile for dynamic recovery. If it is indeed controlled by the same mechanism as the flow stress - the breaking of attractive junctions, for example - then one should be able to use this rather well established profile for application to the instantaneous $T$ - and $\dot{\varepsilon}$-dependence of the flow stress, too [18]. The only problem is a reliable determination of the average forward internal stress in the dynamic recovery case. A strictly heuristic assumption, viz. that the local plastic relaxation inside tangled cell walls leads to an internal stress that is always about 0.7 times the mechanical collapse stress, was indeed successful. Together with the applied stress, which is a $\sigma_{\mathrm{v}}$ at this point, this yields the equation 
$g=g_{0}\left[1-\sqrt{0.7+0.3 \frac{\sigma_{\mathrm{v}} / \mu}{\sigma_{\mathrm{vo}} / \mu_{0}}}\right]^{2}$

The exponent $q=2$ is equivalent to a triangular obstacle profile near the top; together with $p=1 / 2$, it gives rise to the profile shown in Fig. 8. Note that the meaning of $g_{0}$ in this profile is changed, it is twice the inverse of the initial decay rate. In the limit of zero stress, the activation energy would be astronomical $\left(37.5 g_{0}\right.$, in excess of $\left.100 \mathrm{eV}\right)$. From this point of view, the intersection profile is quite 'athermal'; but its initial peak nevertheless leads to a measurable $T$ - and $\dot{\varepsilon}$ dependence.

\section{Summary}

It appears that any one mechanism of dislocation/obstacle interaction (which, incidentally, includes the lattice resistance [1]) can be well described by a phenomenological relation of the form

$\Delta G=k T \ln \frac{\dot{\varepsilon}_{0}}{\dot{\varepsilon}}=\mu b^{3} g_{0}\left[1-\left(\frac{\tau / \mu}{\hat{\tau} / \mu_{0}}\right)^{p}\right]^{q}$

where $q$ is typically $3 / 2$ or 2 and $p$ is typically less than 1 ; once these exponents are picked for a particular mechanism, they become constants. The value of $g_{0}$ should be a constant for this mechanism in any material; that of $\hat{\tau}$, on the other hand, may depend on the specific specimen series (including the texture) and on the tensor character of the test being performed.

An equation like 13 links the temperature and strainrate dependencies of the flow stress. The kinetics of strain hardening, which is very important, can be characterized by a scaling stress (such as $\sigma_{\mathrm{v}}$ ) which itself is thermally activated according to Eq. (9) (or Eq. (12)); in this case, it appears that $q=2$ and $\mathrm{p} \approx 1 / 2$.

If allowance is made for an arbitrary (but measured) dependence of the activation energy on stress, a law like Eq. (1) can describe the kinetics of large-strain plasticity over a very large range of strain rates and temperatures (excepting the range in which lattice diffusion is undisputed as the governing mechanism, and any range in which dynamic effects might be important). For a limited, but often practical range, an expansion around a standard state is proposed (Eqs. (7) and (8)).

It may be possible to unify the thermal activation law for the dislocation contribution to the flow stress and the thermal activation law for dynamic recovery (according to Eq. (12)). This approach could be promising; but it requires further study, also in other materials.

The problems of formulating constitutive relations exhibit the hallmarks of modeling (rather than making a theory); use mechanisms that are based on physical insight (which suggest certain dimensionless variableand-parameter combinations), put them in a phenomenologically sound framework (such as using state parameters only) and, most importantly, fit any open parameters or unknown functions to appropriate experiments.

\section{Acknowledgements}

Much of the contents of this paper was developed during the long-term cooperation with Heinz Mecking. The work has been supported by the US Department of Energy Division of Basic Energy Sciences.

\section{References}

[1] U.F. Kocks, A.S. Argon, M.F. Ashby, Prog. Mater. Sci., Pergamon, Oxford, 1975, vol 19.

[2] A.S. Argon (Ed.), Constitutive Equations in Plasticity, MIT Press, Cambridge MA, 1975.

[3] U.F. Kocks, in: A.S. Argon (Ed.), Constitutive Equations in Plasticity, MIT Press, Cambridge, MA, 1975, pp. 81-115.

[4] E.W. Hart, C.Y. Li, H. Yamada, G.L. Wire, in: A.S. Argon (Ed.), Constitutive Equations in Plasticity, MIT Press, Cambridge, MA, 1975, pp. 149-197.

[5] S.R. Chen, U.F. Kocks, in: A.D. Freed, K.P. Walker (Eds.), High-Temperature Constitutive Modeling: Theory and Application, ASME, New York, 1991, pp. 1-12.

[6] H. Mecking, in: A.W. Thompson (Ed.), Work Hardening in Tension and Fatigue, TMS-AIME, Warrendale, PA, 1977, p. 67.

[7] K. Lücke, H. Mecking, in: R.E. Reed-Hill (Ed.), The Inhomogeneity of Plastic Deformation, American Society Metals, Cleveland, 1973, p. 223.

[8] U.F. Kocks, J. Eng. Mater. Tech. 98 (1976) 76.

[9] U.F. Kocks, in: U.F. Kocks, C.N. Tomé, H.-R. Wenk (Eds.), Texture and Anisotropy, Cambridge University Press, Cambridge, 1998 Chapters 8, 9.

[10] P.S. Follansbee, U.F. Kocks, Acta Metall. 36 (1988) 81.

[11] G. Schoeck, A. Seeger, Defects in Crystalline Solids, Physical Society, London, 1954, p. 340.

[12] P. Haasen, Phil. Mag. 3 (1958) 384.

[13] U.F. Kocks, in: J.D. Embury, A.W. Thompson (Eds.), Modelling of Materials Behavior and Design, TMS, Warrendale, PA, 1990, pp. 77-88.

[14] U.F. Kocks, H. Mecking, in: The Physics and Phenomenology of Strain Hardening, 1999, in press.

[15] U.F. Kocks, Dislocations and Properties of Real Materials, Institute of Metals, London, 1985, pp. 125-143.

[16] G. Saada, Acta metall. 8 (1960) 841.

[17] U.F. Kocks, S.R. Chen, Phys. Stat. Sol. (a) 131 (1992) 403.

[18] U.F. Kocks, S.R. Chen, H. Mecking, in: D.S. Wilkinson, J.D Embury (Eds.), Advances in Crystal Plasticity, Can. Inst. Mining, Metallurgy and Petroleum, 1992, pp. 87-112. 